\title{
Association of IgG and IgM antibodies to phenolic glycolipid-1 antigen of Mycobacterium leprae with disease parameters in multibacillary leprosy patients
}

\author{
N F LYONS,* E J SHANNON, $\dagger$ B P B ELLIS* \\ \& B NAAFS $\ddagger$ \\ *University of Zimbabwe, Department of Medical Microbiology, \\ Godfrey Huggins School of Medicine, Harare, Zimbabwe; †Gillis \\ $W$. Long Hansen's Disease Center, Carville, Louisiana, USA; \\ $\ddagger$ Ministry of Health, Harare, Zimbabwe
}

\section{Accepted for publication 30 September 1987}

\begin{abstract}
Summary IgG and IgM antibodies to the phenolic glycolipid-1 (PGL-1) antigen of Mycobacterium leprae were assessed using an enzyme-linked immunosorbent assay (ELISA) in 77 multibacillary leprosy patients. No correlations were found when their absorbance values were compared to: disease type and duration, bacillary load, reactional status, or concurrent secondary infection. A statistical difference was seen between patients with and without neurological deficiency.
\end{abstract}

\section{Introduction}

Cellular immune response to Mycobacterium leprae is under intensive investigation but following purification of antigens and the development of more sensitive techniques interest in humoral response has also risen. The first studies used the cross-reacting antigen 7 in a sophisticated radioimmunoassay. ${ }^{1,2} \mathrm{~A}$ more recent study ${ }^{3}$ demonstrated a unique phenolic glycolipid antigen of $M$. leprae which differs from the glycolipids found in other mycobacteria.

This specificity of PGL-1 of M. leprae has led to the development of the use of ELISA to detect circulating antibody in leprosy patients. ${ }^{46}$ Elevation of PGL-1 IgG and IgM globulin levels occurs in multibacillary leprosy but this is less marked, or absent, in paucibacillary disease.

Considerable variation of globulin levels appears to occur even between patients with the same classification of multibacillary disease. This investigation compares some of the factors which may account for this variation including: disease type and duration, bacillary load, reactional status, concurrent secondary infection and neurological deficiency.

\section{Materials and methods}

Seventy-seven multibacillary leprosy patients were selected for the study, all of whom were either outpatients or short-term inpatients of the Tropical Diseases Unit at Harare Central Hospital, Zimbabwe. 
Disease classification in all cases was based on the Ridley-Jopling? ${ }^{7}$ scheme using clinical and histopathological criteria. The duration of disease ranged from 3 months to 40 years and some patients had previously received monodapsone therapy. All were currently receiving a 2-year regimen of rif ampicin $600 \mathrm{mg}$ and clof azimine $300 \mathrm{mg}$ once monthly and clof azimine $50 \mathrm{mg}$ and dapsone $100 \mathrm{mg}$ daily.

Reactional status was assessed clinically as either reversal reaction (RR) or erythema nodosum leprosum (ENL). Neurological deficit was detected using sensory and voluntary muscle testing. Anaesthesia of hands and feet was assessed with pressure graduated single nylon filaments and by two-point discrimination tests. Cotton wool wisps or a light-pressure nylon filament was used to detect corneal anaesthesia. Motor deficit of the hand was determined by flexion and extension of the wrist and metacarpophalangeal joints and by adduction and abduction of the digits. Plantar flexion and dorsiflexion, tarsal inversion and eversion and flexion and extension of metatarsal-phalangeal joints were used to determine similar motor deficit of the feet. Facial nerve involvement was assessed by weakness of facial musculature including blink and eye closure.

Serum was separated from whole blood and stored, without preservative, at $-20^{\circ} \mathrm{C}$ until use. Specimens were thawed once only for the purpose of this investigation.

The ELISA used was basically that of an earlier study ${ }^{8}$ as modified for use with PGL- $1 .{ }^{5}$ Specifically PGL-1 (kindly provided by Dr P Brennan) was mixed with $0.05 \mathrm{M}$ carbonate buffer $\mathrm{pH}$ $9 \cdot 2$ and suspended by sonication with a probe at 45 Watts for 90 s (Ultrasonics, Inc., Plainville, NY, USA).

The suspended antigen was diluted in the same buffer to a concentration of $2 \mu \mathrm{g}$ in $50 \mu \mathrm{l}$, the volume which was added to 48 wells of a 96-well polyvinyl flatbottom microtitre plate (Cook Labs., Alexandria, Va, USA). The remaining 48 wells received $50 \mu \mathrm{l}$ of the carbonate buffer without antigen.

The plates were covered with a plastic lid and incubated overnight at $4^{\circ} \mathrm{C}$. After incubation, the wells were washed 3 times for 7 min each with $200 \mu$ l of wash buffer consisting of $0.01 \mathrm{M}$ phosphate buffered saline (PBS) pH 7.2 containing 1\% bovine serum albumin (Fraction V, Sigma, St Louis, Mo, USA). After washing, the wells were blocked by the addition of $100 \mu \mathrm{l}$ of $0.01 \mathrm{M} \mathrm{PBS}-\mathrm{pH} 7 \cdot 4$ containing $5 \% \mathrm{BSA}$ and incubated at $37^{\circ} \mathrm{C}$ for $1 \mathrm{~h}$.

The wells were then washed as before and $50 \mu \mathrm{l}$ of each serum sample, diluted 1:300 in wash buffer, was added to quadruplicate wells of the microtitre plate: 2 with antigen and 2 not containing antigen. Each plate also contained negative controls (pooled serum samples from two normal individuals) (Mean \pm SD Delta Abs. for $\operatorname{IgG}=0 \cdot 134 \pm 0 \cdot 043 ; \operatorname{IgM}=0.012 \pm 0.014, \mathrm{~N}=72$ ); positive multibacillary controls (pooled serum samples from one polar lepromatous and one subpolar lepromatous patient), (Mean \pm SD Delta Abs. for $\operatorname{IgG}=0.314 \pm 0 \cdot 065 ; \operatorname{IgM}=0.077 \pm 0.032$, $\mathrm{N}=72$ ); and positive paucibacillary controls (serum samples from two borderline tuberculoid patients) (Mean \pm SD Delta Abs. for $\mathrm{IgG}=0 \cdot 159 \pm 0 \cdot 072$; $\mathrm{IgM}=0.045 \pm 0 \cdot 020, \mathrm{~N}=72$ ). The plates were incubated at $37^{\circ} \mathrm{C}$ for $45 \mathrm{~min}$, removed and washed as before.

Horseradish peroxidase conjugated IgG fraction of goat antihuman IgG ( $\gamma$-chain specific) and horseradish peroxidase conjugated IgG fraction of goat antihuman IgM ( $\mu$-chain specific) (Cappel Labs., Cochranville, Pa, USA) were diluted in wash buffer. Fifty microlitres of the peroxidaselinked goat antiserum to human IgG and IgM heavy chain each containing $35 \mathrm{ng}$ of the precipitating antibody was added to quadruplicate wells of the microtitre plate: 2 with antigen and having been incubated with serum and 2 without antigen and having been incubated with serum.

The plates were incubated for $45 \mathrm{~min}$ at $37^{\circ} \mathrm{C}$ when they were removed and washed as before. After washing, $50 \mu \mathrm{l}$ of a solution containing $0.4 \mathrm{mg} / \mathrm{ml}$ orthophenylene-diamine (Sigma, St Louis, Mo, USA) with $0.002 \% \mathrm{H}_{2} \mathrm{O}_{2}$ in $0.02 \mathrm{M}$ sodium acetate buffer $\mathrm{pH} 5.5$ was added to each well. The plates were incubated at room temperature for $10 \mathrm{~min}$ when the reactions were stopped by adding 5 $\mathrm{N} \mathrm{HCl}-50 \mu \mathrm{l}$ per well.

Absorbances were read at $492 \mathrm{~nm}$ with a spectrophotometer (Titertek Multiscan, Flow Labs., Richmond, Va, USA). Mean absorbance values and coefficient of variation for each set (pair) of like 
wells were calculated. If the coefficient of variation for either set exceeded $20 \%$, values were not taken and tests were repeated. The antibody reactivity to PGL-1 for each serum sample was calculated by subtracting the mean absorbance of duplicate samples in the carbonate buffer coated wells from the mean of duplicates of the PGL-1 coated wells.

\section{Results}

Absorbance values for IgG and IgM globulins to PGL-1 are presented in Figures 1-6. The duration of disease (Figure 3(a) and 3(b)) is calculated from the year of onset indicated by the patients and are not necessarily the same as the onset of treatment.

Patients with neural deficit resulting from known episodes of R R are excluded from Figure 4. At the time when blood specimens were taken 14 patients were experiencing RR and 22 had ENL (Figure 5). Eighteen patients had secondary infection at the time the specimen was taken or within 2 months previously (Figure 6).

\section{Discussion}

The presence of PGL-1 antibody in sera of leprosy patients is a specific marker of the disease. The ELISA provides a convenient method of assessing antibodies to PGL-1 and with caref ul internal controls is suitable for handling large numbers of specimens. The method is relatively inexpensive and can be incorporated easily into leprosy control programmes. Although the methodology can be

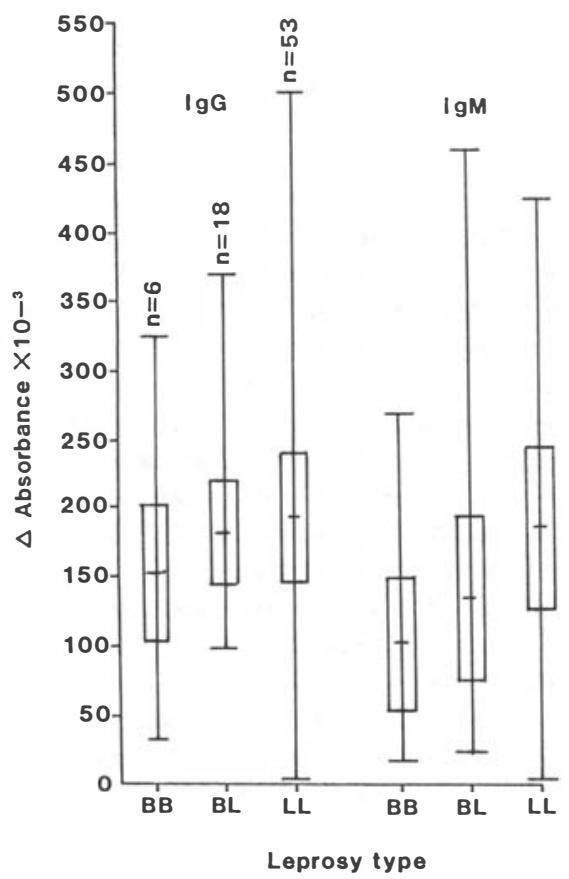

Figure 1. IgG and IgM absorbance values and leprosy classification. (The range of results is indicated by the vertical line, the standard deviation by the vertical column and the mean by the horizontal bar.) 


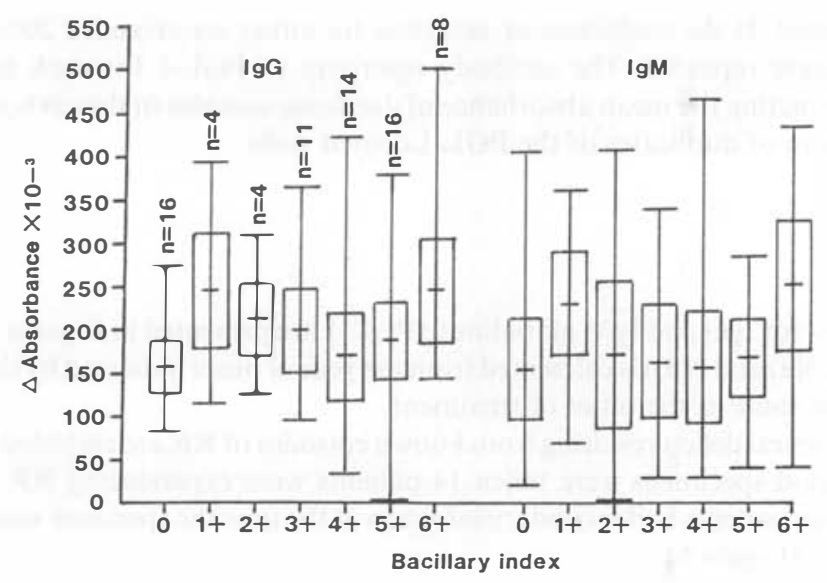

Figure 2. IgG and IgM absorbance values and bacillary index (assessed by slit skin ear smears). The BI of 4 cases was not recorded at the time blood was collected.

standardized, in our experience there is a wide variation in the $\operatorname{IgG}$ and $\operatorname{IgM}$ absorbance values to PGL-1 from patients within the lepromatous spectrum. This observation is in agreement with previously reported findings among LL patients. ${ }^{4}$

Good correlations with parameters including clinical classification and bacterial indices (BI) with IgM absorbance values to the deacylated PGL- $1^{6}$ or the native PGL-19,10 have been reported. The results presented here are in contrast to these observations. While showing an upward trend in IgG and IgM absorbance values from mid-borderline to lepromatous, the wide scatter and overlap indicated a marked lack of correlation. A similar wide deviation and lack of correlation was seen in absorbance values of IgG and IgM antibodies to PGL-1 and BI. This observation was in agreement with those of a previous study. ${ }^{11}$ They found no significant correlation between IgG, IgM and IgA absorbance values to PGL-1 and BI among lepromatous patients.

Clearly there are distinct differences among laboratories, especially when patients at the lepromatous end of the spectrum have been assayed for their antibody responses to PGL-1. Such opposing findings could be explained by variations in methodology, reagents and experimental design.

This ELISA methodology required the use of secondary antibodies. We attempted to standardize our methodology by using equal ng of precipitating peroxidase conjugated anti- $\mu$ and anti- $\gamma$ reagents. However, the equalization of the number of peroxidase molecules conjugated to the antiglobulins, their capacity and affinity to react with the two heavy chains of IgG and the 10 heavy chains of $\operatorname{IgM}$ cannot be standardized. Furthermore it is not clear whether discontinuous solid phase assays like the ELISA which require frequent washing steps measure antibody concentration of affinity. ${ }^{12}$ The enormous range of responses to this $M$. leprae-specific antigen is not easy to explain. It seems that the humoral immune response to $M$. leprae antigens varies between patients but individually is remarkably stable. ${ }^{13}$ Individual response may be mediated by hereditary factors and contact with environmental mycobacterial antigens. ${ }^{14} \mathrm{~A}$ strong response to other mycobacterial antigens may interfere with the stimulating effect of glycolipids on the humoral immune system.

In addition the presentation of the PGL-1 antigen could be masked. In vitro the lipid content of the whole native antigen might block binding sites on the ELISA, although this may not be the case in vivo where recognition and humoral response may depend on exposed saccharide precursors. If this were the case it could explain the lack of a significant rise in antibody level from $1+$ to $6+$ 

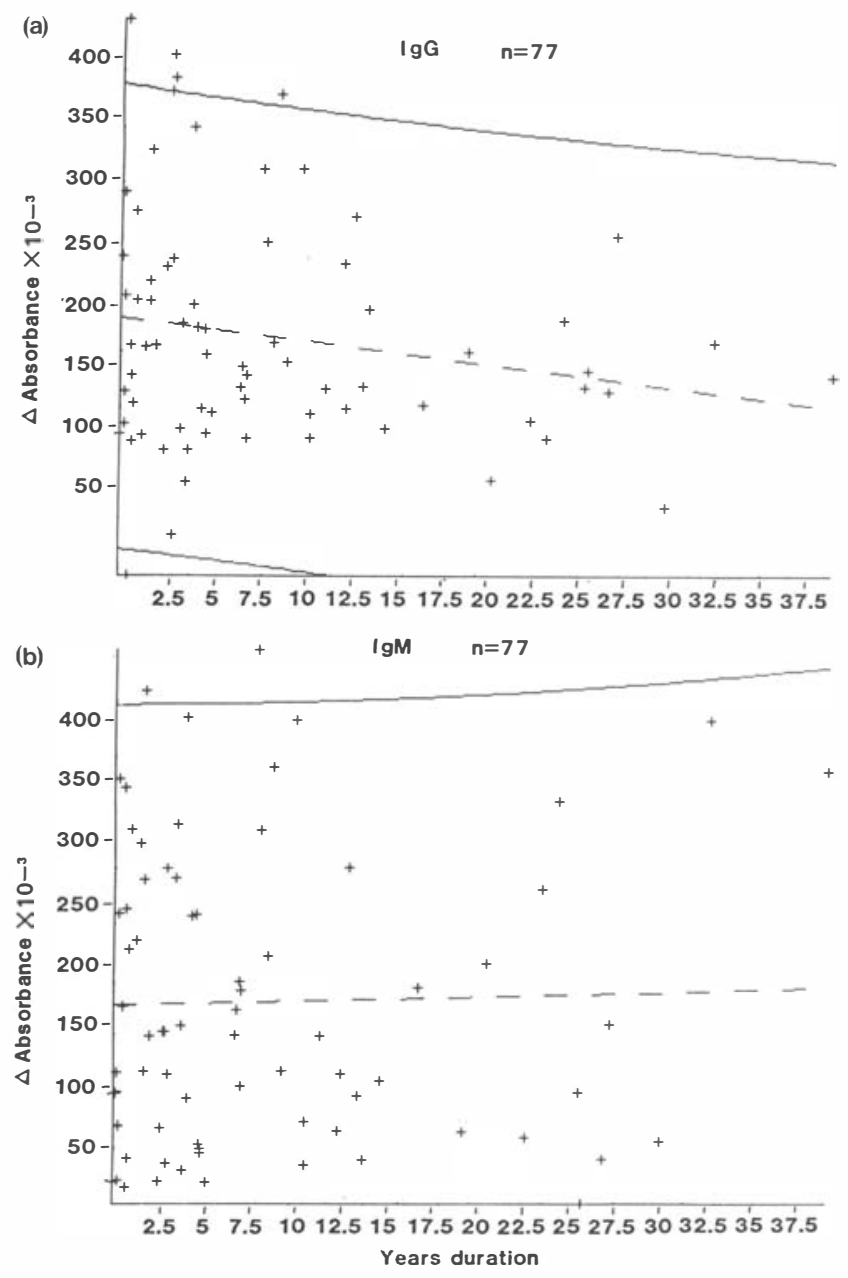

Figure 3(a). IgG absorbance values and disease duration. The central dotted line indicates the regression line interval (solid bounded by the $95 \%$ confidence line). (b) IgM levels and disease duration.

bacteriological index as a significant level of lipid is found in the skin of multibacillary leprosy patients. ${ }^{15}$

Duration of disease also appears to have little eff ect on absorbance values for IgG and IgM antiPGL-1 immunoglobulins although there is a suggestion in the results presented here that IgG levels fall faster than IgM. This is in agreement with observations made by Melson for whole M. leprae antigen. ${ }^{16}$ Chronicity of these levels might be explained by the enormous load of antigen present in multibacillary cases which must take many years to clear.

If neurological deficity can be taken as evidence of progression through the higher resistance paucibacillary forms of the disease then absence of such trauma might indicate onset nearer the lepromatous pole. Comparison of IgG and IgM absorbance values to PGL-1 showed that patients presenting neural deficit had significantly lower IgM absorbance values than patients presenting 


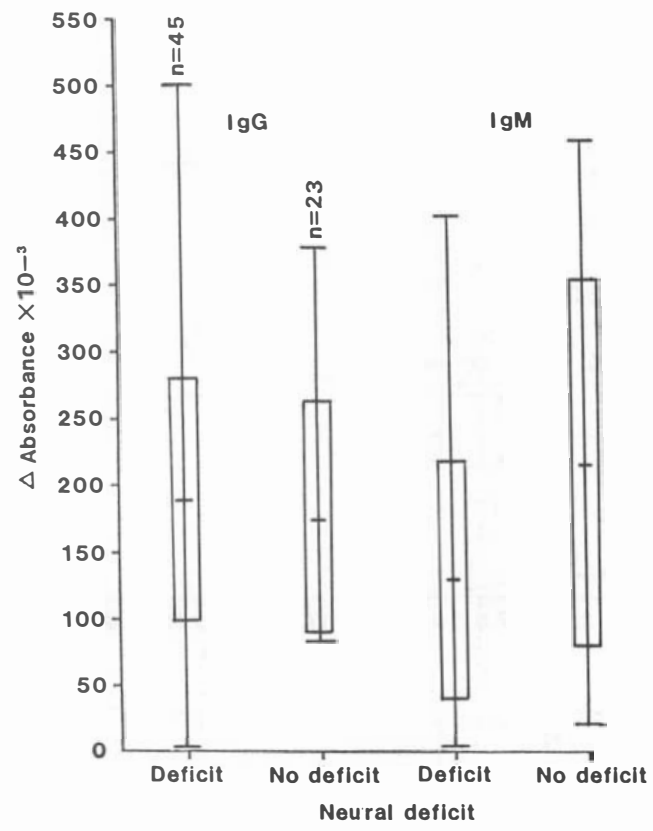

Figure 4. IgG and IgM absorbance values of patients with and without neural deficit. (Nine patients with neural deficit known to be due to RR were omitted.)

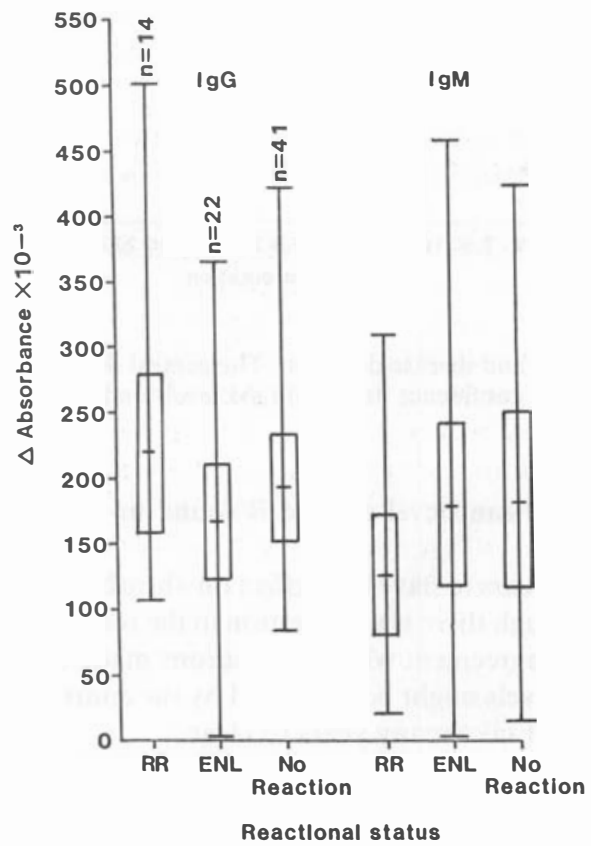

Figure 5. IgG and IgM absorbance values in RR, ENL and non-reactional patients. 


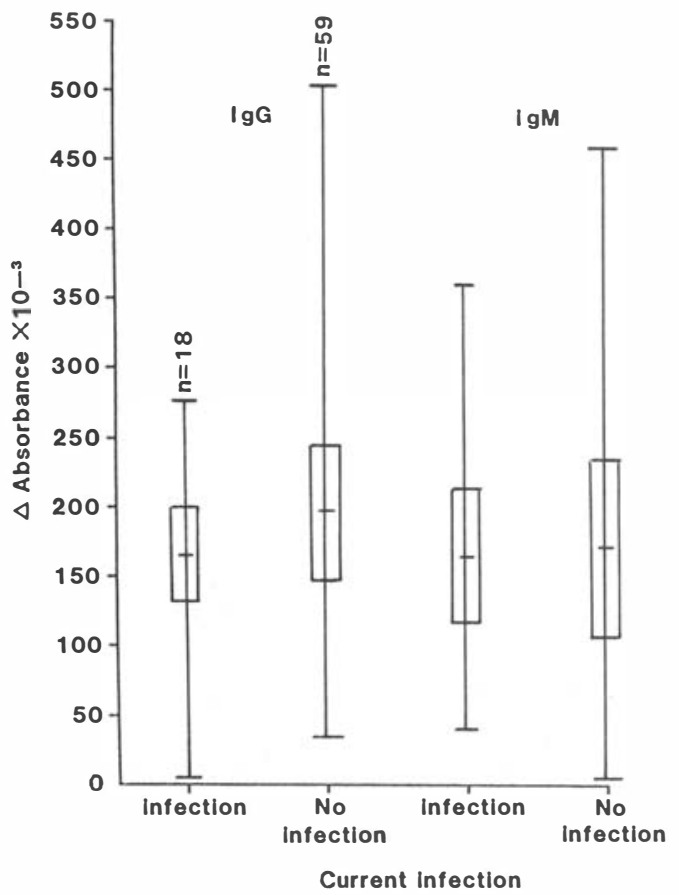

Figure 6. IgG and IgM abosrbance values in patients with and without concurrent secondary infections.

with no neural deficit (Students $t$-test, $P<0 \cdot 02$ ). There was no significant difference with IgG antiPGL-1 absorbance values and neural deficit.

Reactional status appears not to bias IgG and IgM immune response to PGL-1 although the mean IgG absorbance values were higher than IgM in RR. This was not seen in patients presenting with ENL.

Melson et al. ${ }^{16}$ and Cruickshank \& Ellis ${ }^{17}$ found an increase in immunoglobulin during ENL. These investigators however, followed individual patients during the course of the reactional episodes and were not assessing a single serum specimen as in our series.

Many leprosy patientsexhibit a secondary inf ection but no evidence of nonspecific polyclonallyinduced antibodies produced as the result of such infection was seen in this investigation.

From the results obtained it appears that detection of immunoglobulins against native PGL-1 antigen is of limited value in differentiating between the multibacillary forms of leprosy. With the exception of neurological deficit other factors including bacillary load, duration of disease, reactional status and secondary infections seem not to influence the IgG and IgM absorbance values to PGL-1.

\section{Acknowledgments}

We would like to thank Mrs $\mathbf{J}$ Els for her technical assistance, Mr M Mabena for preparing the figures and Mrs M Masterman for typing the manuscript.

The leprosy programme in Zimbabwe is supported by the Associazione Italiana Amici Di Raoul Follereau and the Leprosy Mission. 


\section{References}

${ }^{1}$ Melsom R, Naafs B, Harboe M, Closs O. Antibody activity against Mycobacterium leprae antigen 7 during the first years of DDS treatment in lepromatous (BL-LL) leprosy. Lepr Rev, 1978; 49: 17-29.

2 Yoder L, Naafs B, Harboe M, Bjune G. Antibody activity against Mycobacterium leprae antigen 7 in leprosy: Studies on variation in antibody content throughout the spectrum and on the effect of DDS treatment and relapse in BT leprosy. Lepr Rev, 1979; 50: 113-21.

${ }^{3}$ Hunter SW, Brennan RJ. A novel phenolic glycolipid from Mycobacterium leprae possibly involved in immunogenicity and pathogenicity. $J$ Bact, 1981; 147: 728-35.

${ }^{4}$ Brett SJ, Draper P, Payne SN, Rees DJW. Serological reactivity of a characteristic phenolic glycolipid from M ycobacterium leprae in sera from patients with leprosy and tuberculosis. Clin Exp Imm, 1983; 52: 271-9.

${ }^{5}$ Cho SN, Yaragihara DL, Hunter SW, Gelber RH, Brennan PJ. Serological specificity of phenolic glycolipid1 from Mycobacterium leprae and its use in the serodiagnosis of leprosy. Infect Imm, 1983; 41: 1077-83.

${ }^{6}$ Young DB, Buchanan TM. A serologic test for leprosy with a glycolipid specific for Mycobacterium leprae. Science, 1983; 221: 1057-9.

7 Ridley DS, Jopling WH. Classification of leprosy according to immunity. A five-group system. Int J Lepr, 1966; 34: 255-73.

${ }^{8}$ Voler A, Bidwell D, Bartlett A. Microplate enzyme immunoassays for the immunodiagnosis of virus infections. Manual of Clinical Immunology. Washington, DC: American Society for Microbiology, 1976.

${ }^{9}$ Bach MA, Wallach D, Hoffenbach BFA, Cottenot F. Antibodies to phenolic glycolipid-1 and to whole M ycobacterium leprae in leprosy patients: Evolution during therapy. Int J Lepr, 1986; 54: 256-67.

${ }^{10}$ Levis WR, Meeker HC, Schuller-Levis G, Sersen E, Schwerer B. IgM and IgG antibodies to phenolic glycolipid-1 from Mycobacterium in leprosy: Insight into patient monitoring, erythema nodosum leprosum, and bacillary persistence. J Invest Derm, 1986; 86: 529-34.

1 Truman RW, Shannon EJ, Hastings RC. Host responses to the phenolic glycolipid-1 antigen of M. leprae. Int $J$ Lepr, 1985; 53: 710-11.

12 Steward MW, Lew AM. The importance of antibody affinity in the performance of immunoassays for antibody. J Imm Meth, 1985; 78: 173-90.

${ }^{13}$ Bjorvatn B, Naafs B, Kronvall G. Stability of individual antimycobacterial precipitation patterns during treatment for lepromatous leprosy. Int J Lepr, 1978; 46: 144-8.

${ }^{14}$ Lyons NF, Naafs HB. The influence of environmental mycobacteria on the prevalence of leprosy clinical type. Int J Lepr, 1987; 55: in press.

15 Young DB. Detection of mycobacterial lipids in skin biopsies from leprosy patients. Int J Lepr, 1981; 49: 198204.

${ }^{16}$ Melson R, Harboe M, Naafs B. Class specific anti-Mycobacterium leprae antibody assay in lepromatous leprosy (BL-LL) patients during the first two to four years of DDS treatment. Int J Lepr, 1982; 50: 271-81.

${ }_{17}$ Cruickshank JG, Ellis BPB. Leprosy and the serodiagnostic test for tuberculosis. J Clin Path, 1977; 30: 95-8. 